\title{
AI Based Rescue Robot : A Literature Survey
}

\author{
D.O.I - 10.51201/Jusst12485 \\ http://doi.org/10.51201/Jusst12485 \\ Dr Mrudula Nimbarte, Associate Professor \\ CSE Department JD COEM, Nagpur, India. \\ Madhulika Wannewar, Varsha Pandhare, Kshitij Choubey, Vipul Gajbhiye, Vaibhav Pandey \\ IT Department JD COEM, Nagpur, India.
}

\begin{abstract}
According to the statistics on numbers and rates of fire disaster in world, millions of lives of peoples, animals and on- duty fire fighter are lost every year. It gives the high demand for an autonomous machine to operate the fire disaster rescue operation efficiently and help to fire fighter team. The proposed paper involves the design of a robot that can analysis the situation at victim place and send the full report to the server of fire fighter team which can help them to deal with the disaster situation. Robot also aids the fire fighter team to search the victim and rescue them to safe places. The robot will not only be localize itself but also aids to turn off the fire. The Robot will help to rescue peoples and animals in fire disaster in efficient manner with least harm to rescue team.
\end{abstract}

Keywords-AI, sensors, Automation, TensorFlow, OpenCV

\section{INTRODUCTION}

Disaster causes can be classified into two main groups-natural and man-made. In the past, pro-disaster rescue work was performed by human where machine assistance was not effectively utilized as it was not improved enough. As the time passed, the unprecedented number and scales of natural and humaninduced disasters has urged the emergency search and rescue community around the world to seek for newer, more effective equipment to enhance their efficiency. Rely on search dogs, camera mounted probes and human help is still considered as the assisting tools and technology for rescue as well as search purposes. But, with the advancement of science and technology, intelligent robots equipped with advanced sensors and detectors are attracting more and more attentions from researchers and rescuers. The developed countries though achieved the technology and went on production for rescue robots, in case of developing or underdeveloped countries, this advanced and intelligent assisting tool remains a dream todate. This paper illustrates how to develop the technology of a rescue robot within a limited budget or low-cost in a developing country as well as effectiveness of reusable materials in making this rescue robot.

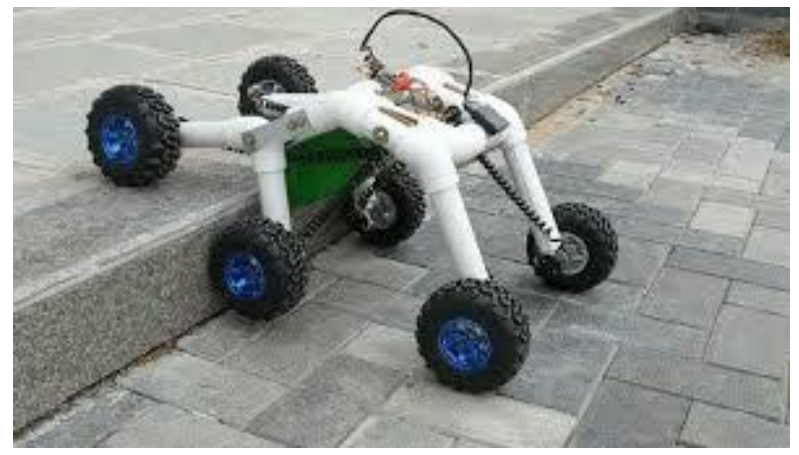

Fig.1. Outer structure

\section{RELATED WORK}

In [1] "Highly Automated Vehicles and Self-Driving cars", authors discussed the design and development of unmanned vehicles which includes robotic and automation studies. As we aim to develop AI-based automatic self-decision making rescue robot adaptive in any earth surface, this paper helps a lot. Here the author explains about how the increasing computational efficiency in image data processing and sophisticated algorithms, helps robotic vision is to take self-decision and analyze the real-time situation. Components of Self-Driving Development: - "algorithms", "development tools", "hardware". Algorithms should satisfy the requirements of three main tasks of the self-driving that is perception, localization, and planning. One of these is the application of the end-to-end black-box learning algorithm by observation is that the vehicle learns how to navigate, negotiate, and adjust control signals by observing stimulus. Development tools should provide a platform for the Data handling (data collection, labelling, generation and enhancement, processing and sensor calibration), Algorithm support (a flexible training environment for AI algorithms and high-level sensor fusion), Testing (algorithms verification, false rejection). In hardware, the author discusses 
various sensors as camera, lidars, ultrasonic with two approaches: camera first approach and lidars as primary sensor approach. Here name of some open-source platforms also given such as "Udacity" for building and training of selfdriving vehicles. Advanced open-source frameworks for complex software and algorithms implementation are "Comma.ai" and "Baidu". Frameworks (among the most popular are Caffe, Theano, Torch, and TensorFlow, OpenCV) generally support fully connected, convolutional, and even recurrent Neural Network architectures.

In [2] "A New Multi-Bug Path Planning Algorithm for Robot Navigation in Known Environments", authors discussed the multi-bug algorithm which is used for path planning and obstacle avoiding. As we are developing rescue robot which can tackle any obstacle which comes in the path while moving toward the destination point, this paper helps a lot. Here the author explained that how they used multi-bug algorithm for reaching the destination point in lesser run time. In multi bug path planning, a bug is virtually moved toward the destination point and while moving if any obstacle is encountered then the algorithm generates two virtual bugs which move along the boundary of the object in different direction and once the obstacle is tackled successfully then the shortest path is chosen which gives the best path as output. In multi bug planning algorithm, it assigns state value to different states using distance transform method. Distance transform helps in finding the distance from every pixel value to the closest non-zero pixel and it also helps in detecting corners or edges in an image. According to the algorithm the robot can switch between online and offline mode. If there are changes in the environment then it switches to online mode so that user could guide it. Multi bug path planning algorithm works efficiently in known environment but in case of unknown environment it fails to give good results. As in unknown environment if some obstacle is encountered then it cannot move in both the direction at the same time because it virtually creates two bugs which move along the boundary for finding the best path with optimal time.

In [3] paper, authors described about how sensors are used in tracking the target. It is also being observed that rescue system must be able to locate quickly the victims within the search space. Past Several years, have shown great advance in the capabilities of wireless sensors. We can easily search the victims by using wireless sensors present in the robot. Robotic Sensors are developed working in Sensor Networks as well as Mobile Networks like robots can easily carry larger loads and collect dense measurements. They can also interact with the stationary sensors by improving the connectivity. In mobile networks, small sensing devices move in a space over time to monitor physical and environmental conditions.

Authors also explained about Gradient Propagation algorithm which will run on all the nodes present. It guarantees about each sensors individual target, gradient value will cover minimum number of hops between the sensors and the one detecting a target (victim), conditions are given to see when the target is visible to the network. Also explained about robot architecture which has three states, in which in first step, it finds the target after in second robot finds another target and at the last robot losses the target signal.

In [4] paper the human being detected by using live body sensor. It's an embedded application made by using ZIGBEE. Zigbee basically work as mediator between robot and control unit. The Author says that the human body emits the thermal radiation it received and manipulated the RIP sensor. The author told that the thermal signals from the RIP sensor sending to the microcontroller and that microcontroller digitize these signal and send to the zigbee. They used the GSM for wireless system for the efficient communication. It also uses the GUI based software that records the data. This project helps to save human lives by using this autonomous vehicle during the earthquake disaster.

In [5] paper, Since past few years Deep learning with Neural Networks has gained a vast structure and influence on the world.Some of the most used popular techniques for image recognition and object detection are Region-based Convolutional Neural Networks (RCNN), Faster-RCNN, Single Shot Detector (SSD) and You Only Look Once (YOLO). In this paper, an SSD and Mobile Nets based algorithms are implemented for detection and tracking inpython environment. SSD object detection composed of two parts,first the extraction feature map and second applying convolution filters to detect objects. Mobile Net is a Neural Network which is used for classification and recognition and SSD is a framework which is used for multibox detection. Object detection involves detecting region of interest of object from given class of image. Different methods are-Frame differencing, Optical flow, Background subtraction. SSD have shown results with considerable confidence level.

This model showed excellent detection and tracking results on the object trained and can further utilized in specific scenarios to detect, track and respond to the particular targeted objects in the video surveillance. This real time analysis of the ecosystem can yield great results by enabling security, order and utility for any enterprise.

\section{PROPOSED METHODOLOGY}

Figure 2 contains the structure of the system proposed in this paper.

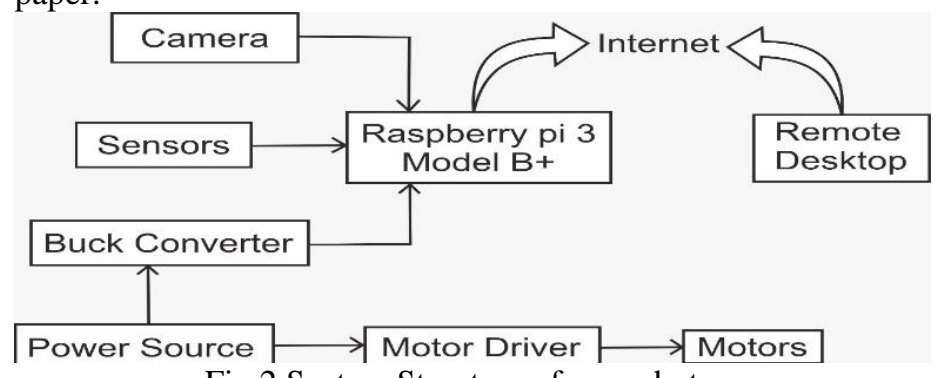

Fig.2.System Structure of our robot

The main components of the system are:

i. Raspberry pi $3 \mathrm{~B}+$ :

In this project Raspberry $\mathrm{Pi} 3 \mathrm{~B}+$ is used as a microprocessor. In Raspberry $\mathrm{Pi} 3 \mathrm{~B}+\mathrm{BCM} 2837 \mathrm{~B} 0$ is present as system on chip (SOP); it works more efficiently and faster than Raspber- 
ry Pi 2. The CPU is having 64-bit quad core processor which works at $1.4 \mathrm{GHz}$ speed, which is more than Raspberry Pi 3 as its speed is $1.2 \mathrm{GHz}$. It also contains $1 \mathrm{~GB}$ RAM (LPDDR2 SDRAM), On-board wireless LAN, On-board Bluetooth with 4.2 HS low-energy (BLE), 4 x USB 2.0 ports, Ethernet with $300 \mathrm{Mbit} / \mathrm{s}$ and Full size HDMI 1.3a port. It also includes camera interface (CSI), Display interface (DSI) and microSD card slot inserted with a memory card.The Graphical Processing Unit (GPU) is clocked at 400MHz/300MHz.

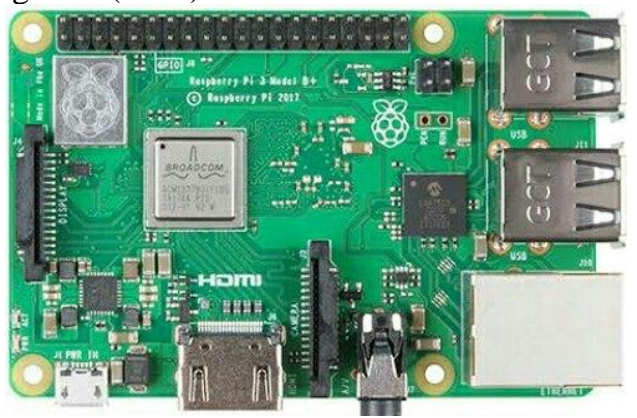

Fig.3. Raspberry Pie 3b+

ii. GSM module:

In this project global system for mobile communication (GSM) network is used to avoid limitation regarding the range and dual tone multiple frequencies (DTMF) function of a cell phone is used to control the functions of the robot. It gives us flexibility so that we can control the robot from wide range also. Different codded signals are generated for communicating between the communication port of computer and the GSM module of the robot. All these commands or signals are transmitted wirelessly to GSM module of the robot.

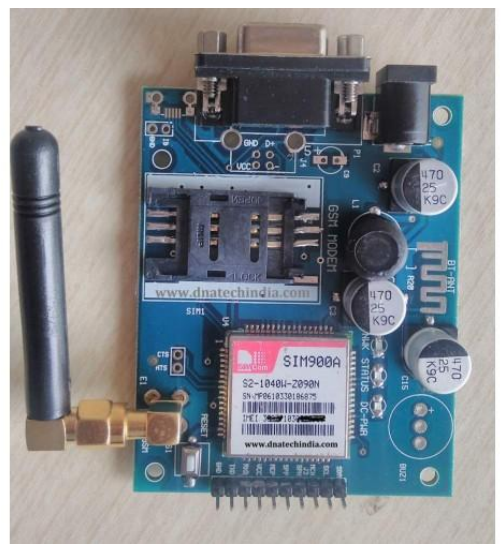

Fig.4. GSM Module

\section{iii. Camera:}

In this project camera module is used for capturing and monitoring live video stream. It is also used to detect motion, objects and for gathering various important data regarding the environment. The data which is gathered from the camera can help in various ways such as in analyzing the situation where the robot is currently present. Camera module plays a very important role as so many important operations depend on it such as object detection and various image processing operations.

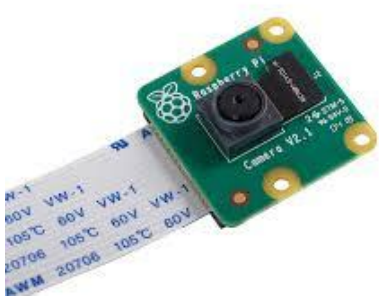

Fig.5.Camera

iv. PIR SENSORS:

It is called as Passive Infrared Sensors. We can detect humans easily using these PIR sensors. As the sensors range is less, the sensor is mounted to a robot that can move automatically. PIR Sensors detect victims (target) within the distance of $10 \mathrm{~m}-12 \mathrm{~m}$. As human body emits infrared waves and this waves are detected by the PIR Sensors and give output in high Quality. They work better in daylight and won't have light sensors to disable them during the daytime.

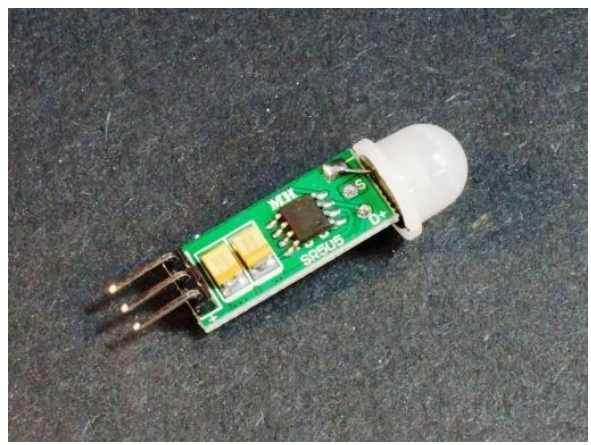

Fig.6. PIR Sensor

\section{v. SERVO MOTORS:}

Servo motors are used in robotics in order to activate movements giving arms to its precise angle. It also controls the wheels of robots; it also produces torque to move, start and stop the vehicle and control its speed.

Servo motors are of three types: Positional Rotation servo Motor, Continuous Rotation Servo Motor and Linear Servo Motors.

Positional Rotation Servo Motor: It is the most common type of servo motors. It includes physical stops located in the gear mechanism to stop turning outside these limits to guard the rotation sensor. This includes aircraft, robots, toys and many other applications.

Continuous Rotation Servo Motor: It can go in any direction indefinitely. This type of motor is used in RADAR dish if you are riding one on a robot or you can use one as a drive motor on a mobile robot.

Linear Servo Motor: It is similar with the positional rotation servo motor. These are not simple to find, but sometimes we can find them at hobby stores where they are used as actuators in higher models like airplanes. 


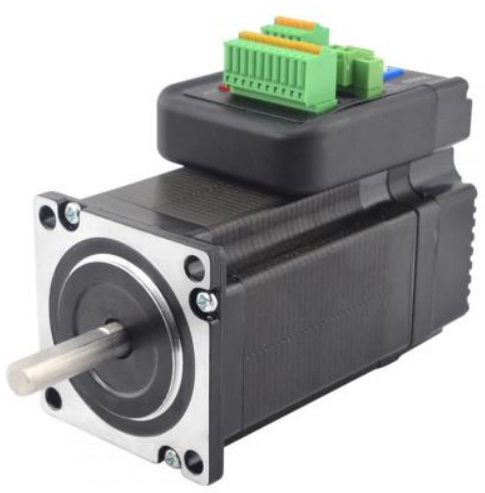

Fig.7.Servo motor

\section{vi. INFRARED SENSORS:}

These sensors are basically used to see the obstacles. This sensor works the same way as the animal echolocation (location of object by reflected sound ex. Dolphins, bat, etc.) Robot sends out a sound signal or a beam of infrared light and detects the signal's reflection. It basically detects heat released by an object (can be any object present in the environment) as well as detects the motion.

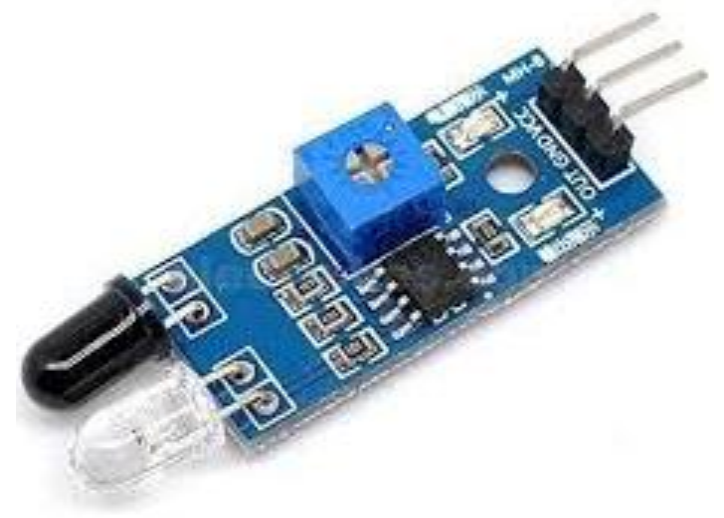

Fig.8. Infrared Sensors

\section{CONCLUSION}

Robotics develops man made mechanical devices that can be moved automatically or with help of remote controls.
Robotics technology is already in use in various different sectors like industrial, transportation, healthcare etc. The goal of robotic technology is to broaden the use and the effectiveness of the robots in various different fields. Robots are developed to perform multiple activities at a time for the welfare of the human being in the most integrated and planned manner by enhancing productivity and quality. As for handling the firefighting situations robots can be a good option for reducing life casualty. As we are always putting others life on risk so for handling and analyzing the situation robots can be used as a replacement.

\section{References}

[1] Árpád Takács, Imre Rudas, Dominik Bösl, and Tamás Haidegger, "Highly Automated Vehicles and Self-Driving Cars"- IEEE robotics \& automation magazine: December 2018.

[2] Bhanu Chander V, Asokan T and Ravindran B, "A New Multi-Bug Path Planning Algorithm for Robot Navigation in Known Environments", 2016.

[3] Joshua Reich and Elizabeth Sklar, "Robot-Sensor Networks for Search and Rescue", Research Gate Paper 2006.

[4] Purnima G, Asst. Prof. Aravind S,Renju Mary Varghese, Neethu Anna Mathew, Gayathri C S, "Alive Human Body Detection and Tracking System Using an Autonomous Pc Controlled Rescue Robot", IJETAE 2015.

[5] Chandan G, Ayush Jain, Harsh Jain, Mohana, "Real Time Object Detection and Tracking Using Deep Learning and OpenCV", 2018.

[6] Matsuno.F., and Tadokoro,S.(n.d.).Rescue Robots Systems in Japan. IEEE International Conference on Robotics and Biometics.2008.

[7] Xin Chen,Jing Li, "Research on an Efficient Single-Stage Multi Object Detection Algorithm”,2019.

[8] Shigeru Kobayashi 1, Yasuhiro Kobayashi 2, Yoshihiro Yamamoto 3, Taro Watasue 4, Yoshikazu Ohtsubo 5, Takumi Inoue 1, Masanobu Yasuda 2, Toshi Takamori 6 " Development of a Door Opening System on Rescue Robot for Search "UMRS-2007".

[9] Michael Sighenza, Diego Guillen,Dante Arroyo, "Mobile Robots Development: A Case Study from Robotics Competitions and Course Projects".2017.

[10] GUODONG ZHAI, WENTAO ZHANG, WENYUAN HU, AND ZHENDONG JI,"Coal Mine Rescue Robots Based on Binocular Vision: A Review of the State of the Art",2020. 